\title{
Equivalent-neighbor Potts models in two dimensions
}

\author{
Xiaofeng Qian, ${ }^{1}$ Youjin Deng, ${ }^{2,3,}{ }^{*}$ Yuhai Liu, ${ }^{4}$ Wenan Guo, ${ }^{4,5}$ and Henk W. J. Blöte ${ }^{1}$ \\ ${ }^{1}$ Lorentz Institute, Leiden University, P. O. Box 9506, 2300 RA Leiden, The Netherlands \\ ${ }^{2}$ Hefei National Laboratory for Physical Sciences at Microscale, Department of Modern Physics, \\ University of Science and Technology of China, Hefei 230027, China \\ ${ }^{3}$ State Key Laboratory of Theoretical Physics, Institute of Theoretical Physics, Chinese Academy of Sciences, Beijing 100190, China \\ ${ }^{4}$ Physics Department, Beijing Normal University, Beijing 100875, China \\ ${ }^{5}$ Beijing Computational Science Research Center, Beijing 100193, China
}

(Received 24 August 2016; published 1 November 2016)

\begin{abstract}
We investigate the two-dimensional $q=3$ and 4 Potts models with a variable interaction range by means of Monte Carlo simulations. We locate the phase transitions for several interaction ranges as expressed by the number $z$ of equivalent neighbors. For not-too-large $z$, the transitions fit well in the universality classes of the short-range Potts models. However, at longer ranges, the transitions become discontinuous. For $q=3$ we locate a tricritical point separating the continuous and discontinuous transitions near $z=80$, and a critical fixed point between $z=8$ and 12. For $q=4$ the transition becomes discontinuous for $z>16$. The scaling behavior of the $q=4$ model with $z=16$ approximates that of the $q=4$ merged critical-tricritical fixed point predicted by the renormalization scenario.
\end{abstract}

DOI: 10.1103/PhysRevE.94.052103

\section{INTRODUCTION}

In phase transitions, the range of the interactions plays an important role. Models with interactions decaying as a negative power $-p$ of the distance appear to display a considerable variety of universality classes as a function of $p$ and as a function of the dimensionality [1-3]. For large $p$ the interactions decay fast and one finds the usual short-range universal behavior. For sufficiently small $p$ the interactions decay only slowly and one finds mean-field-like critical behavior. For intermediate values of $p$ the critical exponents may depend continuously on $p$.

A different way to modify the range of the interactions is specified in the so-called equivalent-neighbor models, in which the pair interactions are constant up to a range $R$ and then abruptly fall to zero. Following Ref. [4], we refer to these models as medium-range models. In the limit $R \rightarrow \infty$, the equivalent-neighbor model reduces to the mean-field model; for sufficiently small $R$ it will naturally display the usual short-range universal behavior. But it seems that the analogy with the case of power-law decay of interactions ends here. Medium-range Ising models, with interactions of a variable range $R$, display uniform crossover from the vicinity of the mean-field fixed point at $R=\infty$ to the Ising critical fixed point at small $R$ [4]. The model belongs to the Ising universality class for all finite $R$. The scaling field parametrizing the crossover phenomenon is the irrelevant Ising temperature field. The flow diagram for the Ising model is shown in Fig. 1.

The question naturally arises whether such a uniform crossover also occurs in the more general context of the $q$-state Potts model [5], of which the Ising model is the special case with $q=2$. Another possibility is suggested by the renormalization scenario for the two-dimensional dilute $q$-state Potts model [6] with $0<q<4$. In this context, the leading irrelevant field, parametrizing the critical surface in

*Corresponding author: yjdeng@ustc.edu.cn parameter space, is controlled by the activity of the vacancies. When the latter parameter is increased, while adjusting the Potts coupling to maintain criticality, a threshold occurs where the model becomes tricritical. Beyond the threshold the ordering transition becomes discontinuous. If the parameters controlling the irrelevant fields of the dilute and the equivalentneighbor Potts models are sufficiently analogous for $q>2$, then this scenario, i.e., a tricritical point separating a range of critical and a range of first-order transitions, might also occur for the equivalent-neighbor Potts model. This is not a new idea. It was already raised by Hilhorst [7] in a discussion following the renormalization results for the Potts model with vacancies [6].

This possibility is also in line with work of Biskup et al. [8] which concerns $q=3$ models with interactions whose strength decays smoothly to zero at infinite range. For a sufficiently slow decay, a first-order transition is predicted. It is also in line with results of Gobron and Merola [9] for the mean-field Potts model perturbed with a Kac potential. In order to provide quantitative answers to the similar question for a simple Potts model system, we consider the equivalent-neighbor model with a finite but variable interaction range $R$, described by the reduced Hamiltonian

$$
\mathcal{H} / k_{\mathrm{B}} T=-K \sum_{i<j} \theta\left(R-r_{i j}\right) \delta_{\sigma_{i}, \sigma_{j}} \quad\left(\sigma_{i}=1, \ldots, q\right),
$$

where the Potts variables $\sigma_{i}$ carry indices that refer to the sites of a square lattice with periodic boundary conditions. Interacting pairs of sites are selected by the step function $\theta$ [defined by $\theta(x)=1$ for $x \geqslant 0$ and $\theta(x)=0$ for $x<0$ ]. Thus, interactions of strength $K$ occur with all neighbors within a distance $R$. In this work, we specify the interaction range $R \propto z^{1 / d}$ by the number $z$ of equivalent neighbors interacting with a spin on a $d$-dimensional lattice.

In particular, we investigate the $q=3$ and the $q=4$ Potts model on $L \times L$ square lattices for a sequence of finite sizes $L$. This task is performed numerically by means of a cluster Monte Carlo method [10] that is especially suitable for this 


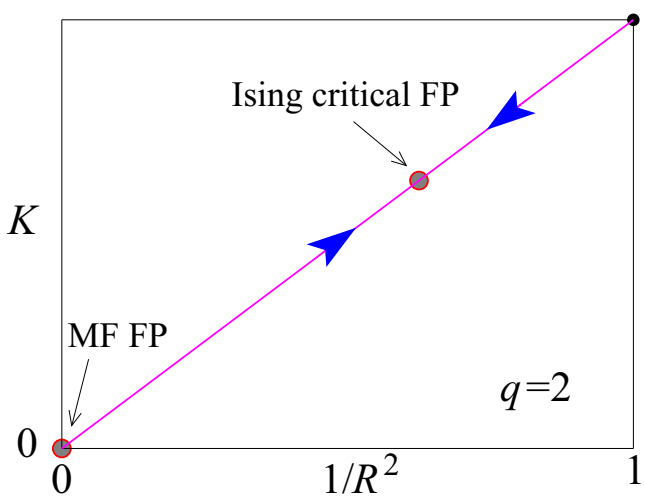

FIG. 1. Schematic renormalization flow diagram along the line of phase transitions of the $q=2$ Potts model with medium-range interactions. The critical line connecting the mean-field (MF) fixed point and the Ising fixed point is parametrized by the range $R$ of the interactions. The finiteness of the interaction range is relevant at the MF fixed point and leads to crossover to the Ising fixed point.

problem, because it not only reduces critical slowing down, but it also remains very efficient for systems with interactions of a long range. During the simulations, we sampled the densities $\rho_{i}$ of the Potts variables in state $i$, from which we obtained the magnetization moments and the Binder ratio [11], as explained in Sec. II. We use finite-size scaling [12] to analyze these data to obtain the location of the phase transitions and their universality classes. In Sec. III we show the results for the $q=3$ Potts model for several values of $z$ in the range $4<z<120$. Results for several $q=4$ Potts models with $4<z<60$ are presented in Sec. IV. Finally, discussions and conclusions are listed in Sec. V. The main results of the present article appeared earlier in the Ph.D. thesis of one of us [13].

\section{METHODS AND SAMPLED QUANTITIES}

The principle of the Monte Carlo technique employed for the study of the present two-dimensional medium-range Potts models was explained in detail in Ref. [10] for the Ising case $q=2$, and it can be trivially generalized to $q>2$ Potts models. The algorithm is organized such that it requires computer time that is almost independent of the number $z$ of interacting neighbors per spin. We used the Wolff-like single-cluster version [14] of the algorithm.

Since the locations of the phase transitions are unknown for general $z$, our first task is to determine them. This determination is based on the Monte Carlo sampling of the moments of the magnetization density $m$. This quantity depends on the densities $\rho_{i}$ of the Potts states $i=1,2, \ldots, q$ as

$$
m^{2} \equiv \frac{1}{q-1} \sum_{i=1}^{q-1} \sum_{j=i+1}^{q}\left(\rho_{i}-\rho_{j}\right)^{2}
$$

This definition is in accordance with the interpretation of the Potts spins as vectors with $q$ equivalent orientations in $q-1$-dimensional spin space. The magnetization moments determine a dimensionless ratio $Q$, related to the Binder cumulant [11], defined as:

$$
Q=\frac{\left\langle m^{2}\right\rangle^{2}}{\left\langle m^{4}\right\rangle} .
$$

The expected finite-size scaling behavior of $Q$ near the transition point is obtained by expansion of the scaling formula for the free energy near the pertinent critical or tricritical fixed point. This leads to

$$
\begin{aligned}
Q(K, L)= & Q_{0}+\sum_{k} a_{k}\left(K-K_{\mathrm{c}}\right)^{k} L^{k y_{t}}+\sum_{j} b_{j} L^{y_{j}} \\
& +c\left(K-K_{\mathrm{c}}\right) L^{y_{t}+y_{1}}+d\left(K-K_{\mathrm{c}}\right)^{2} L^{y_{t}}+\cdots,
\end{aligned}
$$

where $Q_{0}$ is a universal constant, $y_{t}$ is the renormalization exponent describing the scaling of the temperature field, and the $y_{j}$ with $j=1,2, \ldots$ are negative exponents describing corrections that will be discussed later, and the $a_{k}$, the $b_{j}$, $c$, and $d$ are unknown amplitudes. The term with amplitude $d$ describes the nonlinearity of the temperature field as a function of $K$.

In the case of the four-state Potts model, the behavior is less simple because of the presence of a marginal operator, as predicted by the renormalization scenario due to Nienhuis et al. [6]. From a further analysis of the renormalization equations [15-17], it is possible to predict the finite-size scaling behavior of the singular part of free energy as a function of the temperature scaling field $t \simeq K-K_{\mathrm{c}}$, the magnetic scaling field $h$, and the marginal field $v$ as

$$
f_{s}\left(t, h, v, L^{-1}\right)=L^{-d} f_{s}\left(L^{y_{t}} u^{3 / 4} t, L^{y_{h}} u^{1 / 16} h, u v, 1\right),
$$

where $u(L) \equiv 1 /[1-(v / \pi) \ln L]$. Since the magnetization moments can be expressed in terms of derivatives of the free energy with respect to the magnetic field, one can also obtain the expected scaling behavior of $Q$. In leading orders, one finds that, for $K=K_{\mathrm{c}}$,

$$
\begin{aligned}
Q\left(K_{\mathrm{c}}, L\right)= & Q_{0}+c_{1} /(1-b \ln L)+c_{2} /(1-b \ln L)^{2} \\
& +c_{3} /(1-b \ln L)^{3}+\sum_{j} b_{j} L^{y_{j}}+\cdots,
\end{aligned}
$$

where $b \propto v$, and $c_{k} \propto v^{k}$, thus $b \propto c_{1}$ as well. The proportionality constants are universal but unknown. The finite-size scaling behavior of $Q$ near the transition point follows by additional differentiation of $f_{s}$ to the temperature field as

$$
\begin{aligned}
Q(K, L)= & Q\left(K_{\mathrm{c}}, L\right)+\sum_{k} q_{k}\left(K-K_{\mathrm{c}}\right)^{k} u^{3 k / 4} L^{k y_{t}} \\
& +\sum_{j} b_{j} L^{y_{j}}+\cdots,
\end{aligned}
$$

where $q_{k} \propto v^{3 k / 4}$, with universal but unknown proportionality constants.

The ratio $Q$ is a useful quantity to locate phase transitions and to determine the associated temperature-like exponent. From Eq. (4) one finds that the $Q$ versus $K$ curves for different values of the finite-size parameter $L$ intersect at values approaching $K=K_{\mathrm{c}}$ for large $L$. Moreover, the slopes of these curves are asymptotically proportional to $L^{y_{t}}$, which thus allows the estimation of $y_{t}$. 


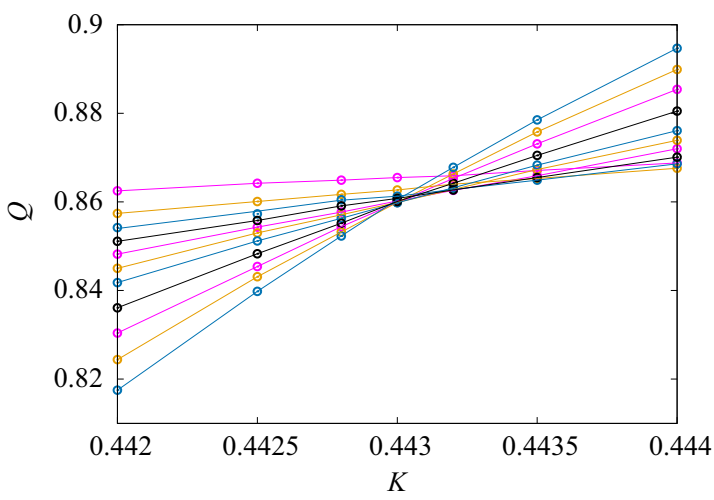

FIG. 2. The Binder ratio $Q$ of the three-state Potts model with eight equivalent interacting neighbors vs. coupling $K$ for system sizes $L=6,9,12,15,18,21,24,30,36,42$, and 48. Larger system sizes correspond with steeper curves.

For each model, cluster simulations [10,14,18] were performed for several system sizes in a suitable range of $K$ near criticality, and $6 \times 10^{6}$ or more samples were taken for each data point specified by $q, K$, and $z$. The intersections of finitesize data for $Q$ versus $K$, taken at different values of $L$ but for the same $q$ and $z$, reveal the location of the critical point. This is illustrated in Fig. 2 for the $q=3$ Potts model with $z=8$, i.e., nearest- and next-nearest-neighbor interactions. A more accurate location was determined by a least-squares analysis according to Eq. (4). Similar analyses were performed for the other choices of $q$ and $z$ investigated in the present work.

We have also searched for possible evidence, in the form of hysteresis loops, for first-order transitions at a finite interaction range. We thus determined the behavior of the energy and the magnetization while the coupling $K$ was changed in small steps separated by long intervals. Furthermore, we investigated the autocorrelation time $\tau$ and the distributions $p(E)$ of the energy and $p(m)$ of the magnetization.

\section{RESULTS FOR THREE-STATE POTTS MODELS}

\section{A. Location and nature of the phase transitions}

The Binder ratio $Q$ is assumed to be universal for critical Potts models with the same $q$, but this universal number still depends on the geometry of the finite system. The relevant factors are the ratio of the microscopic couplings in different directions, the boundary conditions, and the shape of the system, for instance, the aspect ratio of a rectangular periodic box. In this work, we restrict ourselves to systems with square symmetry, which pertains to the lattice, the couplings and to the boundary conditions. The universal value of $Q_{0}$ can therefore conveniently be determined from the nearest-neighbor Potts model, for which we know the exact critical point as $K_{\mathrm{c}}=\ln (1+\sqrt{3})$. We therefore simulated the nearest-neighbor three-state Potts model at the critical point, using square systems with sizes $L=6,7,8, \ldots, 280,320$. We fitted the finite-size data by Eq. (4), using the known values of the critical point and the critical exponents [19], of which the temperature exponent is $y_{t}=6 / 5$ and the leading irrelevant exponent $y_{1}=-4 / 5$. This leads to $Q_{0}=0.85410$ (10).

As a consistency check, we also simulated the dilute Potts model to determine $Q_{0}$ for the three-state Potts model near the critical fixed point, which is located [20] at $K_{\text {cfp }}=1.16941(2)$, $D_{\text {cfp }}=1.376483(5)$. At the critical fixed point, the leading correction term with exponent $-4 / 5$ is suppressed. For the model at the critical fixed point, we simulated 21 systems $L=6,8,10, \ldots, 150,210$, and obtain $Q_{0}=0.85408$ (7) and $y_{1}=-1.13(4)$. This value is close to an expected correction exponent $X_{h 1}-X_{h 2}=-6 / 5$. The values of the magnetic exponents $X_{h 1}$ and $X_{h 2}$ are given in Ref. [19]. When we fix the correction exponent at the value $y_{1}=-6 / 5$, we obtain $Q_{0}=0.85412(5)$. These relatively accurate results for $Q_{0}$ will be useful for the analysis of models with more neighbors.

We also simulated the dilute Potts model to determine $Q_{0}$ at the tricritical point of the three-state Potts model, which is located [20] near $K_{\mathrm{tfp}}=1.649903, D_{\mathrm{tfp}}=3.152152$. For the tricritical dilute Potts model we used system sizes

TABLE I. Results for the Binder ratio $Q_{0}$ and thermal exponent $y_{t}$ for $q=3$ models for several ranges of interaction. These results are obtained by fits of Eq. (4) to the Monte Carlo data, with all parameters left free, except $K_{\mathrm{c}}$ in the case of the nearest-neighbor model. The tricritical point lies in the neighborhood of $z=80$, because the result for $y_{t}$ is closest to the tricritical value $y_{t}=12 / 7$ for $q=3$. For smaller $z$ the results tend to the critical value $y_{t}=6 / 5$ and for larger $z$ to the discontinuity fixed point value $y_{t}=2$ which applies to first-order transitions. The third column "Ms" lists the number of millions of samples taken per data point. The error estimates between parentheses are based on two standard deviations in the statistical analysis.

\begin{tabular}{lcccccc}
\hline \hline$z$ & $L$ & Ms & $K_{\mathrm{c}}$ & $Q_{0}$ & $y_{t}$ & $y_{1}$ \\
\hline 4 & $6-320$ & 25 & $\ln (1+\sqrt{3})(\mathrm{exact})$ & $0.8542(1)$ & $1.20(3)$ & $-4 / 5$ \\
8 & $6-240$ & 8 & $0.442907(3)$ & $0.8536(8)$ & $1.18(2)$ & $-4 / 5$ \\
12 & $6-240$ & 6 & $0.272027(2)$ & $0.8537(4)$ & $1.197(6)$ & $-4 / 5$ \\
20 & $6-240$ & 8 & $0.154075(2)$ & $0.852(4)$ & $1.19(2)$ & $-6 / 5$ \\
28 & $9-240$ & 8 & $0.106430(2)$ & $0.848(4)$ & $1.15(3)$ & $-6 / 5$ \\
36 & $9-270$ & 8 & $0.081432(2)$ & $0.853(6)$ & $1.18(3)$ & $-4 / 5$ \\
48 & $9-270$ & 8 & $0.060112(2)$ & $0.838(16)$ & $1.24(4)$ & $-4 / 5$ \\
56 & $9-360$ & 10 & $0.051188(2)$ & $0.802(6)$ & $1.36(4)$ & $-4 / 5$ \\
68 & $12-600$ & 11 & $0.0418853(8)$ & $0.773(4)$ & $1.45(4)$ & $-4 / 5$ \\
80 & $12-600$ & 8 & $0.0354315(4)$ & $0.753(2)$ & $1.64(4)$ & $-4 / 5$ \\
100 & $18-160$ & 6 & $0.0282084(4)$ & $0.744(8)$ & $1.98(6)$ & $-4 / 5$ \\
120 & $18-120$ & 6 & $0.0234324(4)$ & $0.754(8)$ & $2.01(5)$ & $-6 / 5$ \\
\hline \hline
\end{tabular}


TABLE II. Transition points $K_{\mathrm{c}}$ for three-state Potts models as determined by least-squares fits with $y_{t}$ fixed at $6 / 5$ for $z<80$ and at $y_{t}=2$ for $z>80$. For $z=80, y_{t}$ was fixed at $12 / 7$ although the data in Table I suggest that the tricritical value of $z$ may be slightly larger than 80 . We fixed $Q_{0}=0.85412$ for $z<80, Q_{0}=0.743$ for $z=80$, and $Q_{0}=0.75$ for $z \geqslant 100$. For $z=4$, we used the exact critical value of $K_{\mathrm{c}}$. The error margins are based on two standard deviations in the statistical analysis.

\begin{tabular}{|c|c|c|c|c|c|c|c|}
\hline$z$ & $L_{\min }$ & $K_{\mathrm{c}}$ & $Q_{0}$ & $y_{t}$ & $y_{1}$ & $y_{2}$ & $b_{1}$ \\
\hline 4 & 6 & $\ln (1+\sqrt{3})($ exact $)$ & 0.85412 & $6 / 5$ & $-4 / 5$ & $-6 / 5$ & $0.148(2)$ \\
\hline 8 & 6 & $0.4429080(10)$ & 0.85412 & $6 / 5$ & $-4 / 5$ & $-6 / 5$ & $0.085(5)$ \\
\hline 12 & 6 & $0.2720275(6)$ & 0.85412 & $6 / 5$ & $-4 / 5$ & $-6 / 5$ & $-0.155(2)$ \\
\hline 20 & 9 & $0.1540760(5)$ & 0.85412 & $6 / 5$ & $-4 / 5$ & $-6 / 5$ & $-0.68(2)$ \\
\hline 28 & 9 & 0.1064309 (4) & 0.85412 & $6 / 5$ & $-4 / 5$ & $-6 / 5$ & $-1.94(5)$ \\
\hline 36 & 9 & $0.0814320(4)$ & 0.85412 & $6 / 5$ & $-4 / 5$ & $-6 / 5$ & $-3.37(5)$ \\
\hline 48 & 9 & $0.0601132(3)$ & 0.85412 & $6 / 5$ & $-4 / 5$ & $-6 / 5$ & $-7.85(7)$ \\
\hline 56 & 12 & $0.0511894(2)$ & 0.85412 & $6 / 5$ & $-4 / 5$ & $-6 / 5$ & $-14.3(8)$ \\
\hline 68 & 60 & $0.0418858(2)$ & 0.85412 & $6 / 5$ & $-4 / 5$ & $-6 / 5$ & $-40(6)$ \\
\hline 80 & 48 & $0.03543150(6)$ & 0.743 & $12 / 7$ & $-4 / 5$ & -2 & $1.5(4)$ \\
\hline 100 & 18 & $0.0282086(1)$ & $3 / 4$ & 2 & -1 & -2 & $-2.3(5)$ \\
\hline 120 & 18 & $0.0234323(1)$ & $3 / 4$ & 2 & -1 & -2 & $-2.4(4)$ \\
\hline
\end{tabular}

$L=6,8,10, \ldots, 84,108$. Fits with fixed $y_{t}=12 / 7$ [19] and $y_{1}$ left as a free parameter show that there exists a correction to scaling with an exponent near $y_{1}=-1$, with an uncertainty margin of a few tenths. This exponent is consistent with $y_{1}=X_{h 1}-X_{h 2}=-6 / 7$. The $\chi^{2}$ criterion provides strong indications that another correction to scaling is present, but the data are not accurate enough to allow a reliable determination of its exponent. Further corrections may be expected with exponent $y_{2}=-10 / 7$ (irrelevant exponent $X_{14}$ in the Kac table) and with $d-2 y_{h}=-38 / 21$. The resulting values for the $Q_{0}$ are still somewhat dependent on which correction exponent is included. Taking into account the uncertainty due to this dependence, as well as the statistical error margin, the estimated result is $Q_{0}=0.743$ (4).

For several values of $z$, we determine the critical points, and also estimate the temperature exponent by least-squares fits. The results are included in Table I. The dependence of the estimates of $y_{t}$ and of $Q_{0}$ for different $z$ provides some information on the nature of the phase transition. For $z \leqslant 48$, the results are consistent with the universality class of the $q=3$ short-range model. It is, however, clear that crossover phenomena occur near $z=80$, affecting the accuracy of the results and their error estimation. In particular, the results for $y_{t}$ and $Q_{0}$ near $z=56$ display this effect. The results for $z=80$ lie close to the tricritical values given above. For $z>$ 80 the results are consistent with first-order behavior: The value $y_{t}=2$ corresponds with the discontinuity fixed point exponent [21], and the universal ratio is expected to satisfy $Q_{0}=3 / 4$ at the coexistence of three ordered phases and one disordered phase [22]. More accurate estimations of critical points were obtained when the Binder ratio and the temperature exponents were fixed at their expected values. The results are listed in Table II.

In order to shed more light on the crossover phenomenon near $z=80$, we study the first derivative of $Q$ with respect to the coupling $K$ at criticality, which can be derived from Eq. (4) as

$$
\left.\frac{d Q}{d K}\right|_{K_{\mathrm{c}}}=L^{y_{t}}\left(a_{1}+c L^{y_{1}}+\cdots\right),
$$

where $a_{1}$ is the leading amplitude. Only terms of first order in ( $K-K_{\mathrm{c}}$ ) in Eq. (4) survive. From Eq. (8) one finds that, at the transition point,

$$
\frac{\ln (d Q / d K)}{\ln L}=y_{t}+\frac{\ln a_{1}+\left(c / a_{1}\right) L^{y_{1}}+\cdots}{\ln L},
$$

so, since $y_{1}<0$, one expects that a plot of $\ln (d Q / d K) / \ln L$ versus $1 / \ln L$ will yield a straight line for large $L$ with an intercept $y_{t}$ on the vertical axis. The data for $d Q / d K$, as obtained from fits to the $Q$-versus- $K$ simulation results and by numerical differentiation, are shown accordingly in Fig. 3.

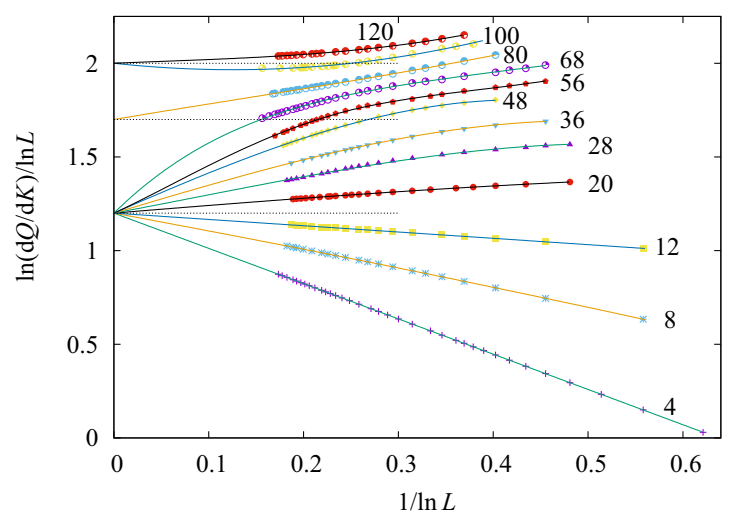

FIG. 3. Finite-size dependence of the derivative of the Binder ratio $Q$ of the three-state Potts model with respect to $K$, taken at $K_{\mathrm{c}}$. The quantity plotted along the vertical scale is defined in the text and chosen such that the data should converge, for sufficiently large $L$, to the temperature exponent $y_{t}$ which is $6 / 5$ for the three-state critical Potts model, 12/7 for the tricritical three-state Potts model, and 2 for the first-order range. These values are shown by thin horizontal lines. The data points for each value of $z$ are connected by a curve which is also intended to guide the eye to the limiting value at $L=\infty$ on the vertical scale, according to our interpretation of the data. The value of $z$ is indicated in the figure for each curve. These results show that the model with $z=80$ lies close to the tricritical point. 


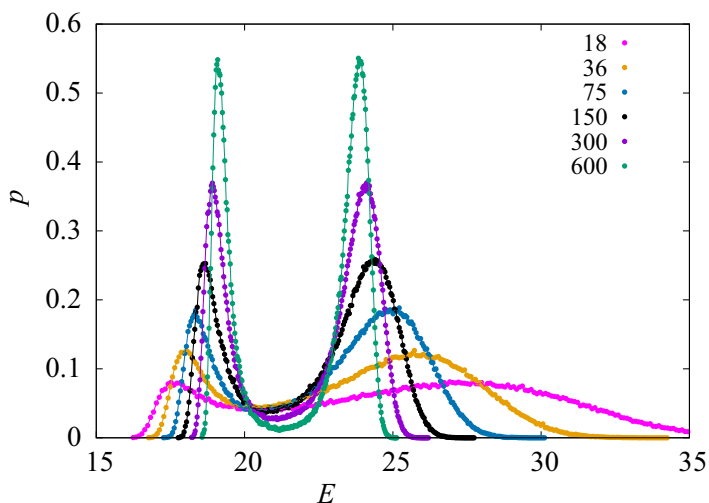

FIG. 4. Histograms of the energy distributions $p$ of finite $q=3$ Potts models with $z=100$ equivalent neighbors. The values of the finite sizes $L$ are shown in the figure. The couplings are chosen such that the two peaks are equally high. These data represent $5 \times 10^{5}$ samples separated by $L / 4$ single-cluster steps per system size, except for $L=600$, where the latter number is $L / 3$.

\section{B. Various results in the first-order range}

We wish to verify the results in the preceding subsection, which indicate that the ordering phase transitions of three-state Potts models with $z \gtrsim 80$ are first order.

\section{Time evolution and histogram}

To display the discontinuous character of the transition in the model with $z=100$ equivalent neighbors, we have recorded the behavior of the energy of an $L=200$ system as a function of Monte Carlo time. The system appears to display a sort of flip-flop behavior between two states with different energies, at random intervals typically in the order of $10^{5}$ Wolff cluster steps. But the fluctuations of the higher-energy state are still considerable, which suggests that we should also bring the aspect of system size into consideration.

Histograms of the energy are shown in Fig. 4 for several system sizes, taken at couplings chosen such that both maxima have the same height. Minor reweighting was applied to this purpose. These results show that the peaks become narrower and the minima between them deeper when the system size increases. This is in accordance with first-order behavior [23].

\section{Hysteresis loops}

We have recorded the behavior of the energy and the magnetization of the model of an $L=600$ system with $z=120$ equivalent neighbors, while the coupling was stepped up or down in small intervals. The results for the energy and the magnetization are displayed in Fig. 5. The energy-like quantity $E$ is defined as the reduced Hamiltonian (1) divided by $-L^{2} K$. These data display clear hysteresis loops. The first-order transition is located near $K_{\mathrm{c}} \approx 0.0234$; this is rather close to the mean-field prediction $[24,25] K_{\mathrm{c}}=0.02310 \ldots$ for $z=120$. The ranges of overlap of the two branches in Fig. 5 are narrow, roughly $10^{-5}$ in $K$. While this is much smaller than the range of metastability according to the mean-field prediction for $q=3$, it is naturally dependent on the system size and the simulation length per data point.

\section{Dynamic behavior}

Figure 6 displays the dynamic behavior of the $q=3$ model with 100 equivalent neighbors at the phase transition point, under single-cluster steps. The figure shows the autocorrelation time $\tau$ versus the system size $L$. The autocorrelation time unit is chosen as the number of Wolff-type single-cluster steps equal to the inverse single-cluster size. In the case of a critical point, one expects $\tau \propto L^{z_{d}}$. The use of logarithmic scales would then lead to a straight line with slope $z_{d}$ if $\tau \propto L^{z_{d}}$ in Fig. 6. The upward curvature of the line indicates that the average cluster size does not scale algebraically with $L$, confirming the weakly first-order character of the transition.

\section{RESULTS FOR FOUR-STATE POTTS MODELS}

\section{A. Auxiliary results}

As for the three-state model, one may attempt to determine the universal ratio $Q_{0}$ from simulations of the nearest-neighbor Potts model at the exactly known critical point. However, the logarithmic corrections for $q=4$ lead to anomalously slow finite-size convergence and inhibit accurate numerical analysis. Instead, we chose the Baxter-Wu model [26], a model of Ising spins on the triangular lattice, with three-spin interactions $K s_{i} s_{j} s_{k}$ in each triangle. It is solved exactly [26] and belongs to the four-state Potts universality class, but without logarithmic corrections. In view of its triangular
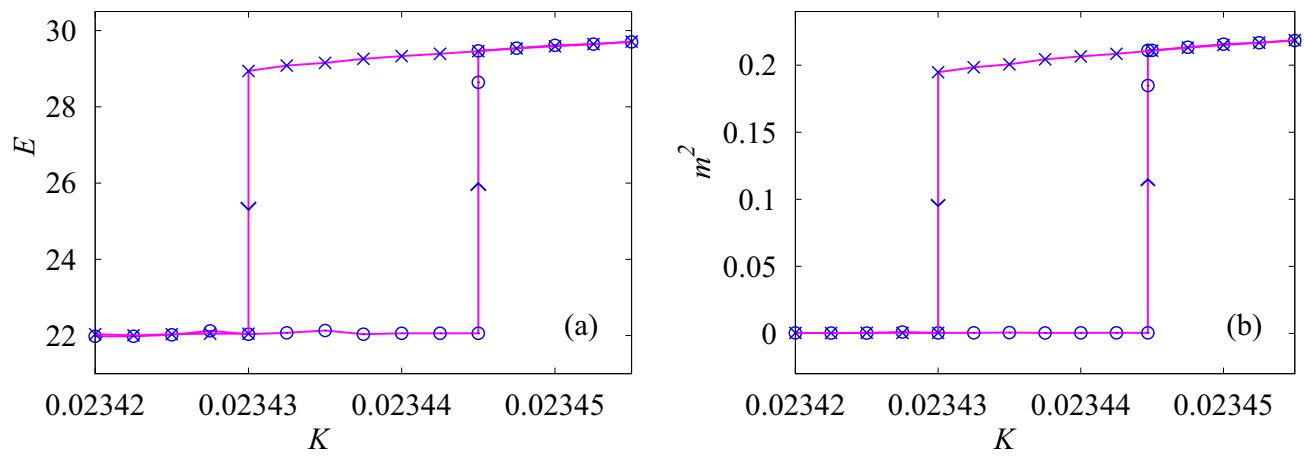

FIG. 5. Hysteresis loops of the energy (a) and the squared magnetization (b) of the $q=3$ Potts model with 120 equivalent neighbors for system size $L=600$. Data points are separated by $2 \times 10^{5}$ single-cluster steps. A data point at the end of the observed metastability could be obtained from intermediate results taken at smaller intervals. 


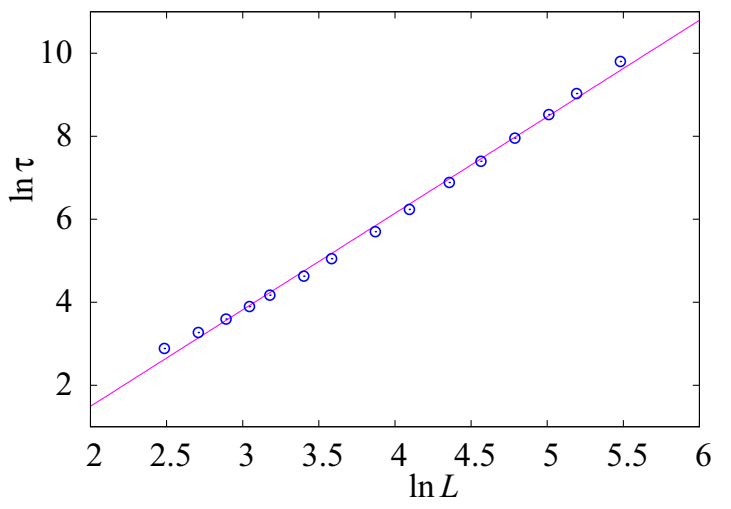

FIG. 6. Dynamic properties of the cluster simulation of the $q=3$ model with $z=100$ equivalent neighbors, in terms of the autocorrelation time $\tau$ versus the system size $L$. The use of logarithmic scales leads to a straight line with slope $z_{d}$ if $\tau \propto L^{z_{d}}$. The upward curvature of the data is in agreement with a weakly first-order transition. The slope of the straight line corresponds to a dynamic exponent $z_{d}=2.3258$. The line is shown for visual aid only.

geometry, caution is needed to obtain the universal result for $Q_{0}$ for models defined on a square periodic box with the proper boundary conditions.

The invariance of boundary conditions under renormalization indicates that value of $Q_{0}$ is universal but still depends on the type of boundary conditions. In the case of periodic boundary conditions, the periodic images may, for instance, form a square or a triangular lattice. It is thus not surprising that the value of $Q_{0}$ was found to differ in these two cases [27]; see also a confirmation by Selke [28] and a discussion by Dohm [29]. Furthermore, in the case that the periodic images form a rectangular pattern, $Q_{0}$ is a universal function of the aspect ratio [27]. In the case of a model with anisotropic couplings, this universal function can be used to determine the equivalent geometric anisotropy ratio [27,30].

In order to account for these effects, we chose the following numerical approach. We simulated Baxter-Wu systems of $L_{x} \times L_{y}$ spins, with $L_{x}$ a multiple of 3 , and $L_{y}$ a multiple of 2 , and $L_{y} / L_{x} \approx 2 / \sqrt{3}$. The $x$ direction is parallel to one set of edges of the lattice. The proper positioning of the periodic box with respect to its periodic images was guaranteed by choosing a square lattice representation of the triangular lattice, with diagonal bonds added in the $(1,1)$ direction in the elementary faces labeled with even $y$, and in the $(-1,1)$ direction in the faces labeled with odd $y$. We employed the Wolff-like variant of an algorithm [31] that freezes one of three sublattices and grows a single Ising cluster on the remaining two sublattices. Simulations, performed at the critical point $K_{\mathrm{c}}=\frac{1}{2} \ln (1+\sqrt{2})$, involved 45 system sizes with $3 \leqslant L_{x} \leqslant 240$, with a number of samples in the order of $10^{9}$ for $L \leqslant 72$ and $10^{8}$ for $L>72$. The periodic boxes defined above are rectangular, with aspect ratios that are only approximately equal to 1 . Therefore, the aspect ratio was included in the fit formula Eq. (4) for the finite-size data as follows:

$$
\begin{aligned}
Q\left(L_{x}, L_{y}\right)= & Q_{0}+b_{1} L^{-1}+b_{2} L^{-7 / 4}+b_{3} L^{-2} \\
& +c_{1} a^{2}\left(L_{x}, L_{y}\right)+\cdots
\end{aligned}
$$

where $L \equiv \sqrt{L_{x} A_{y}}$, with $A_{y} \equiv \sqrt{3 / 4} L_{y}$ the actual size of the rectangular periodic box in the $y$ direction. The aspect ratio is parametrized by $a \equiv\left(L_{x}-A_{y}\right) / \sqrt{L_{x}^{2}+A_{y}^{2}}$. The correction exponent $y_{1}=-1$ was strongly suggested by the finite-size data and is equal to the difference between the first two magnetic exponents $y_{h 2}-y_{h}$ [19]. The exponent $y_{2}=-7 / 4$ is equal to $2-2 y_{h}$ and may arise from the analytic part of the susceptibility. Also, the term with $y_{3}=-2$ helped to reduce the fit residuals, enabling satisfactory fits for $Q$ down to a minimum system size of $L_{x}=6$.

We included an independent determination of $Q_{0}$ from simulations of the dilute $q=4$ Potts model on the square lattice at the estimated fixed point $K_{f}=1.45790, D_{f}=$ 2.478438 , which is very close to the value reported in Ref. [20]. We simulated $L \times L$ systems for a number of finite sizes $L=4,5, \ldots, 80$. Since logarithmic corrections are absent at the fixed point, we used Eq. (10) to fit to the finite-size data but without the term accounting for the aspect ratio. The fits behave very similar to those for the Baxter-Wu model, and the results for $Q_{0}$ of both models agreed satisfactorily.

We performed several other fits, by including a correction with an exponent $y_{2}=-2.5$ instead of -1.75 , and with various subsets of fixed correction exponents. After discarding the fits with a too-large residual $\chi^{2}$, the results are consistent with our final estimate $Q_{0}=0.81505$ (15) where the error estimate is twice the statistical margin of the average of the preferred fits for the two models. This value of $Q_{0}$ will be helpful in the analysis of the results for the $q=4$ equivalent-neighbor models.

In addition to the universal ratio $Q_{0}$, we also investigate the universal ratio $c_{1} / b$ mentioned in Sec. II by means of simulations of a modified Baxter-Wu model. The model remains self-dual when the couplings $K_{\text {up }}$ and $K_{\text {down }}$ in the up- and down-triangles are made to differ. The self-dual line is located at $\sinh 2 K_{\text {up }} \sinh 2 K_{\text {down }}=1$. For $K_{\text {up }} \neq K_{\text {down }}$ the model shifts away from the $q=4$ fixed point and thus acquires logarithmic corrections [32]. The direction of its shift is away from the nearest-neighbor model, into the first-order range. Since the ratio $K_{\text {up }} / K_{\text {down }}$ can be chosen arbitrarily, we can arrange it such that for our range of $L$ values the finite-size-scaling behavior of the model is determined by the renormalization flow in the vicinity of the fixed point. Thus the value of the marginal field $v$ in Eq. (5), as well as that of the parameter $c$ in Eq. (6), remains small. Then, we may assume that higher-order terms with $c_{2}, c_{3}$, etc., in that equation may be neglected. Under this assumption, we attempt to determine the universal ratio $c_{1} / b$ from a fit of Eq. (6) to the Monte Carlo results for $Q$, taken at the self-dual point for a suitable value $K_{\text {up }} / K_{\text {down }}$. We simulated the model with $K_{\text {up }} / K_{\text {down }}=2$ at the self-dual point for 27 system sizes $6 \leqslant L \leqslant 120$. Most of the simulations were rather short, with a few times $10^{7}$ samples, but we also included long runs with about $10^{9}$ samples for $L=24,48$, and 72 . Good statistics is necessary, because the differences of the finite-size data for $Q$ and those of the fixed point, which we wish to analyze, are still quite small. Satisfactory least-squares fits could be obtained on the basis of Eq. (6) for system sizes down to $L_{x}=6$. We obtain $c_{1}=-0.0049(2)$ and $b=0.102(6)$, from which we estimate the universal ratio $c_{1} / b=-0.048$. 
TABLE III. Binder ratio $Q$ and thermal exponent $y_{t}$ as estimated from simulations of the medium-range $q=4$ Potts model. These results suggest that the tricritical point between the critical and firstorder range occurs between $z=12$ and 20. The error margins, quoted as 2 times the standard deviation of the statistical analysis, are not realistic because logarithmic correction factors are omitted in this analysis. Moreover, the errors for $z=60$ may be underestimated because of slow dynamics in the first-order range. The third column shows the number of millions of samples taken for each data point as specified by $K, L$. A number of $K$ values near $K_{\mathrm{c}}$ was chosen for each $L$, typically varying between 6 for $L<20$ and 1 for the largest values of $L$.

\begin{tabular}{lccccccc}
\hline \hline$z$ & $L$ & $\mathrm{Ms}$ & $K_{\mathrm{c}}$ & $Q$ & $y_{t}$ & $y_{1}$ & $y_{2}$ \\
\hline 4 & $12-240$ & 8 & $1.09862(1)$ & $0.840(2)$ & $1.418(5)$ & -1 & $-7 / 4$ \\
8 & $12-224$ & 8 & $0.49098(2)$ & $0.836(2)$ & $1.431(4)$ & -1 & $-7 / 4$ \\
12 & $12-224$ & 30 & $0.30625(2)$ & $0.828(3)$ & $1.490(10)$ & -1 & $-7 / 4$ \\
16 & $12-224$ & 30 & $0.222856(2)$ & $0.814(1)$ & $1.529(10)$ & -1 & $-7 / 4$ \\
20 & $12-224$ & 10 & $0.175842(2)$ & $0.805(1)$ & $1.610(10)$ & -1 & $-7 / 4$ \\
24 & $8-120$ & 12 & $0.144523(2)$ & $0.795(1)$ & $1.70(4)$ & -2 & -3 \\
28 & $8-96$ & 12 & $0.122812(2)$ & $0.788(1)$ & $1.82(6)$ & -2 & -3 \\
36 & $8-84$ & 15 & $0.094528(2)$ & $0.780(1)$ & $1.91(5)$ & -2 & -3 \\
44 & $8-48$ & 25 & $0.076826(4)$ & $0.781(6)$ & $2.02(5)$ & -2 & -3 \\
60 & $12-44$ & 20 & $0.055921(2)$ & $0.804(8)$ & $2.04(5)$ & -2 & -3 \\
\hline \hline
\end{tabular}

\section{B. Critical points}

We estimated the critical points and the temperature exponent $y_{t}$, as well as $Q_{0}$, from the Monte Carlo data for the Binder ratio for several values of $z$ in the $q=4$ medium-range Potts model. As a preliminary analysis, we fitted Eq. (4) to the finite-size data for $Q$, with the values of $Q_{0}$ and $y_{t}$ left free. The correction exponents were fixed at $y_{1}=-1$ and $y_{2}=-7 / 4$. The fit results are shown in Table III. While these results are inaccurate as a measure of the universal quantities, they provide information how the nature of the phase transition depends on $z$. For $z \lesssim 12$, the estimates of $y_{t}$ are smaller than the exact value $y_{t}=3 / 2$, as is usually the case for $q=4$ Potts-like models with short-range interactions [33-35]. The estimates of the Binder ratio are clearly too large in comparison with the universal value $Q_{0}=0.81505(15)$ as listed in Sec. IV A. These discrepancies are explained by logarithmic factors, such as in Eq. (6), which are not taken into account in these fits. These differences decrease when $z$ increases, signaling a decrease of the marginal field $v$. The results for $z \gtrsim 20$ indicate that the model resides in the firstorder range. This is probably also the case for $z=20$, since the $y_{t}$ estimates exceed $3 / 2$, with an increasing trend for large $L$, suggesting crossover to the discontinuity fixed-point value $y_{t}=2$. Since the fixed-point value of $z$ seems to lie between 12 and 20, we have included a model with $z=16$ equivalent neighbors. We realized this by including only four of the eight neighbors at a distance $R=\sqrt{5}$, with coordinates $(x, y)=$ $(2,1),(-1,2),(-2,-1),(1,-2)$. This preserves the fourfold rotational symmetry of the local interacting environment.

We have also determined the first derivative of $Q$ with respect to the coupling $K$ at criticality, similarly as for $q=3$. In the critical range one thus expects, in principle, convergence of $\left(\ln (d Q / d K) / \ln L\right.$ to $y_{t}=3 / 2$, but the presence of a

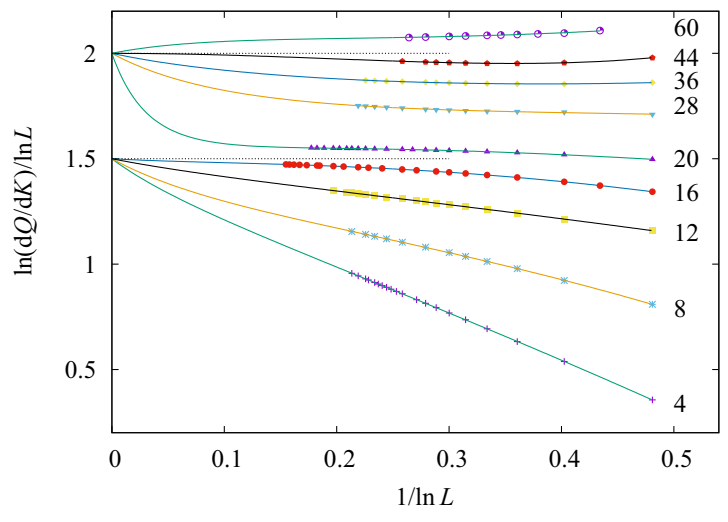

FIG. 7. Finite-size dependence of the derivative at $K_{\mathrm{c}}$ of the Binder ratio $Q$ of the four-state Potts model with respect to $K$. The data points for each value of $z$ are connected by lines which are intended for visual aid and for display of possible extrapolations to $L=\infty$. The values of $z$ are shown in the figure for each curve. This way of plotting should lead, for critical models with sufficiently large $L$, to linear behavior and convergence to the temperature exponent $y_{t}=3 / 2$. However, due to the presence of logarithmic corrections, such behavior may only be observed in practice when the marginal field vanishes. The data in this figure suggest that this is the case near $z=16$.

marginal field leads to corrections behaving as an inverse logarithm of $L$, so the available range of system sizes is insufficient for an accurate result. Nevertheless, the data for $(\ln (d Q / d K) / \ln L$ versus $1 / \ln L$, shown in Fig. 7 are sufficiently clear to demonstrate that the $z=16$ model lies close to the $q=4$ fixed point and signals the boundary between the short-range behavior for $z<16$ and first-order behavior for $z>16$.

In an attempt to obtain more accurate estimates of the critical points, $Q_{0}$ and $y_{t}$ were fixed at their expected values, and a fit formula based on Eq. (7) was used for $z \leqslant 20$. The $z=20$ model still seems to be rather close to the fixed point. However, except for $L=16$, it appears that the accuracy of the fits is limited, because the higher-order logarithmic terms

TABLE IV. Critical points $K_{\mathrm{c}}$ for $q=4$ as derived from fits with the Binder ratio $Q_{0}$ and thermal exponent $y_{t}$ fixed as shown in the table. Error margins are quoted as twice the standard deviation of the statistical analysis. The exponents of the power-law corrections were fixed at the values shown in the table, except for $y_{1}$ in the range $24 \leqslant z \leqslant 44$ where this exponent was left free in the fit.

\begin{tabular}{lccccccc}
\hline \hline$z$ & $L_{\min }$ & $K_{\mathrm{c}}$ & $Q_{0}$ & $y_{t}$ & $y_{1}$ & $y_{2}$ & $\Delta Q(80)$ \\
\hline 4 & 6 & $\ln 3$ (exact) & 0.81505 & $3 / 2$ & -1 & $-7 / 4$ & 0.0346 \\
8 & 12 & $0.49097(1)$ & 0.81505 & $3 / 2$ & -1 & $-7 / 4$ & 0.0245 \\
12 & 8 & $0.306252(1)$ & 0.81505 & $3 / 2$ & -1 & $-7 / 4$ & 0.0136 \\
16 & 12 & $0.222856(1)$ & 0.81505 & $3 / 2$ & -1 & $-7 / 4$ & -0.0010 \\
20 & 12 & $0.175843(1)$ & 0.81505 & $3 / 2$ & -1 & $-7 / 4$ & -0.0072 \\
24 & 8 & $0.144523(1)$ & $4 / 5$ & 2 & $-0.3(1)$ & -2 & -0.005 \\
28 & 8 & $0.122812(1)$ & $4 / 5$ & 2 & $-0.6(1)$ & -2 & -0.010 \\
36 & 8 & $0.094531(2)$ & $4 / 5$ & 2 & $-0.6(1)$ & -2 & -0.018 \\
44 & 8 & $0.076829(2)$ & $4 / 5$ & 2 & $-0.8(1)$ & -2 & -0.012 \\
60 & 12 & $0.055919(2)$ & $4 / 5$ & 2 & -1 & -2 & -0.004 \\
\hline \hline
\end{tabular}



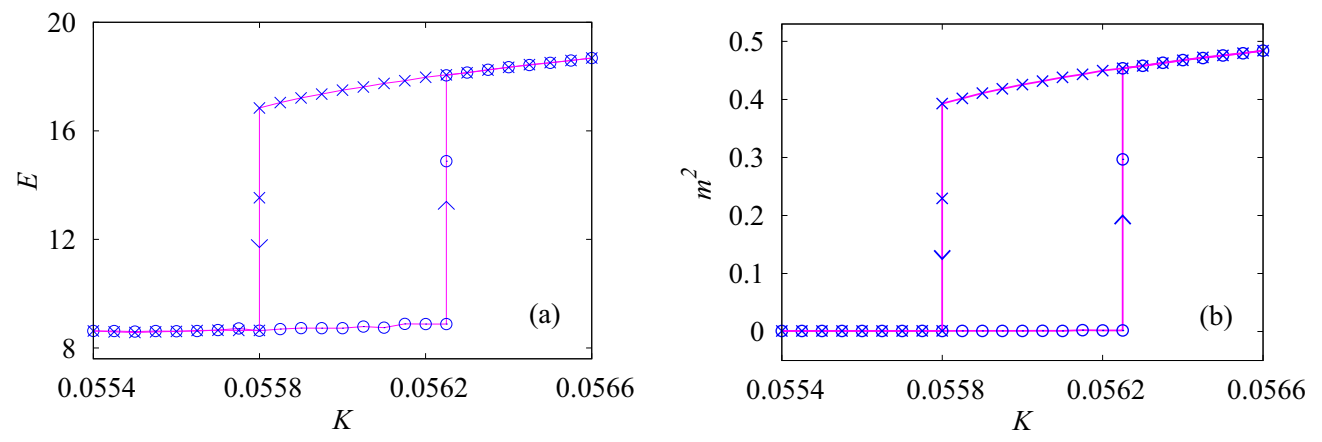

FIG. 8. Hysteresis loops of the energy (a) and the squared magnetization (b) of the $q=4$ model with 60 equivalent neighbors and finite size $L=120$. The energy-like quantity $E$ is equal to the expectation value of the reduced Hamiltonian (1) divided by $-L^{2} K$. Data points are separated by $2 \times 10^{5}$ single-cluster steps. Two data points at the end of the observed metastability could be obtained from intermediate results taken at smaller intervals.

do not converge satisfactorily. As a result, the parameters $b$ and $c_{1}$, purportedly describing the marginal scaling field, are poorly determined. Instead, we define, on the basis of Eqs. (6) and (7), a measure of the marginal field as the sum of the logarithmic terms at $K_{\mathrm{c}}$,

$$
\begin{aligned}
\Delta Q(L) & \equiv \sum_{k} c_{k} /(1-b \ln L)^{k} \\
& =Q\left(K_{\mathrm{c}}, L\right)-\sum_{j} b_{j} L^{y_{j}}-Q_{0} .
\end{aligned}
$$

At a constant finite-size $L$, this quantity represents the scaling function $Q(u)$ as a function of the distance $u$ to the fixed point. Unlike the individual amplitudes, the sum of the logarithmic terms is well determined by the fit, at least within the range of $L$ covered by the data. We chose $L=80$ where the power-law corrections, and their error margins, are relatively small. The results for $\Delta Q(80)$ are included in Table IV. For $z \geqslant 24$ we used a fit formula with a different value $Q_{0}=0.8$ and without logarithmic terms. Power-law corrections are included with exponents as shown in Table IV. The distance to the discontinuity fixed point is purportedly approximated by $\Delta Q(L) \equiv Q\left(K_{\mathrm{c}}, L\right)-Q_{0}$ at a sufficiently large size $L=80$.

The accuracy of the values of $\Delta Q(80)$, shown in the last column of Table IV, is estimated as about 0.001 . The results in the range $4 \leqslant z \leqslant 20$ clearly display a change of sign of the marginal field near $z=16$. The results in the range $24 \leqslant z \leqslant 60$ indicate that the finite-size scaling function of $Q$ describing the crossover from the merged fixed point to the discontinuity fixed point goes through an extremum before approaching the limit $Q=4 / 5$.

\section{Hysteresis loop}

For $q=4$ we have determined the behavior of the energy and the magnetization of an $L=120$ system with $z=60$ equivalent neighbors, while the coupling was stepped up or down in small intervals. We find very clear hysteresis loops, which are displayed in Fig. 8. The first-order transition takes place near $K_{\mathrm{c}}=0.0559$, not far from the mean-field prediction $K_{\mathrm{c}}=0.0593$ for the $q=4$ model with $z=60$ interacting neighbors.

\section{DISCUSSION AND CONCLUSION}

The results in Sec. III indicate that, for the $q=3$ Potts model, the renormalization flow is as shown in Fig. 9 (left-hand side), i.e., the role of the interaction range is similar to that of the fugacity of the vacancies in the dilute Potts model [6]. Our results indicate that the $q=3$ Potts model with $z=80$ lies close to the tricritical fixed point in Fig. 9 and that the critical fixed point corresponds with a value of $z$ between 8 and 12. Also, the results for the $q=4$ model in Sec. IV display this analogy: Increasing the range of the interactions has a similar effect as dilution in the nearest-neighbor $q=4$ Potts model [6,20].

Thus our results are well described by the renormalization flow diagram that follows when the critical and tricritical fixed points in Fig. 9 merge into a single fixed point that is marginally irrelevant on the short-range side and marginally relevant on the long-range side [6], as shown in Fig. 9. Since the marginal field is absent in the Baxter-Wu model [26], that model faithfully reproduces the expected scaling behavior a the merged fixed point. Our results in Sec. IV B indicate that also the $q=4$ Potts model with $z=16$ lies close to the merged fixed point in Fig. 9.

These results for the $q=3$ and 4 Potts model stand in a strong contrast with the Ising case $q=2$, where the effects of vacancies and of an increased interaction range differ. The Ising tricritical point such as present in the dilute Ising model is absent in the $q=2$ model with medium-range interactions [4], as illustrated in Fig. 1. There is only a gradual crossover, with mean-field behavior only in the limit $R \rightarrow \infty$. Ising universality applies for all finite $R$.
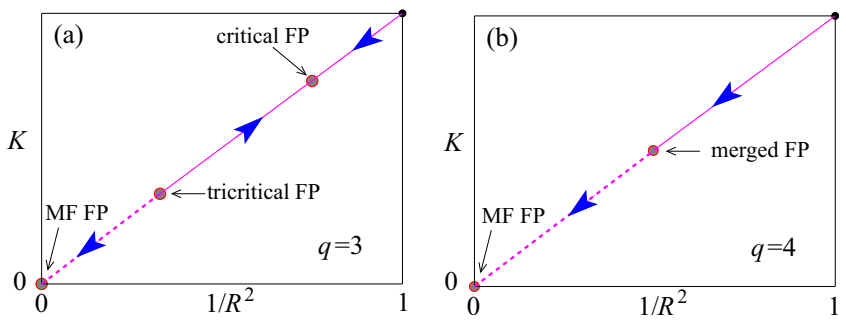

FIG. 9. Renormalization flow of the three-state (a) and of the four-state (b) Potts model along the line of ordering transitions. 
An obvious question not answered in the present work is how the present work can be generalized to nonintegral values of $q$, i.e., the medium-range random-cluster model [36]. Self-consistent solution in the mean-field limit $z \rightarrow \infty$ shows that, for $q<2$, the critical behavior of this model is the same as that of the mean-field percolation model, with critical exponents $\beta=1, \gamma=1$, and $\delta=2$. For this range of $q$, we conjecture that the mean-field fixed point is unstable with respect to finite values of $z$. We thus expect that, for $q<2$, the universal behavior is that of the short-range $q$-state random-cluster model, for all finite ranges $R$ of interaction.

\section{ACKNOWLEDGMENTS}

We acknowledge useful discussions with A. D. Sokal about the value of $Q_{0}$ in the first-order range. H.B. is grateful for the hospitality extended to him by the Faculty of Physics of the Beijing Normal University. This research was supported by the National Natural Science Foundation of China under Grants No. 11275185 (Y.D.) and No. 11175018 (Y.L. and W.G.) and by the Fundamental Research Funds for the Central Universities of the Ministry of Education (China).
[1] M. E. Fisher, S.-K. Ma, and B. G. Nickel, Phys. Rev. Lett. 29, 917 (1972).

[2] J. Sak, Phys. Rev. B 8, 281 (1973).

[3] E. Luijten and H. W. J. Blöte, Phys. Rev. B 56, 8945 (1997); Phys. Rev. Lett. 89, 025703 (2002).

[4] E. Luijten, H. W. J. Blöte, and K. Binder, Phys. Rev. E 54, 4626 (1996).

[5] R. B. Potts, Proc. Cambridge Philos. Soc. 48, 106 (1952).

[6] B. Nienhuis, A. N. Berker, E. K. Riedel, and M. Schick, Phys. Rev. Lett. 43, 737 (1979).

[7] H. J. Hilhorst (private communication, 1980).

[8] M. Biskup, L. Chayes, and N. Crawford, J. Stat. Phys. 122, 1139 (2006).

[9] T. Gobron and I. Merola, J. Stat. Phys. 126, 507 (2007).

[10] E. Luijten and H. W. J. Blöte, Int. J. Mod. Phys. C 6, 359 (1995).

[11] K. Binder, Z. Phys. B 43, 119 (1981).

[12] For a review, see, e.g., M. P. Nightingale, in Finite-Size Scaling and Numerical Simulation of Statistical Systems, edited by V. Privman (World Scientific, Singapore, 1990).

[13] X. Qian, Ph.D. thesis, Scaling, Clusters and Geometry, University of Leiden (2006).

[14] U. Wolff, Phys. Rev. Lett. 60, 1461 (1988).

[15] M. Nauenberg and D. J. Scalapino, Phys. Rev. Lett. 44, 837 (1980).

[16] J. L. Cardy, M. Nauenberg, and D. J. Scalapino, Phys. Rev. B 22, 2560 (1980).

[17] J. Salas and A. D. Sokal, J. Stat. Phys. 88, 567 (1997).

[18] R. H. Swendsen and J. S. Wang, Phys. Rev. Lett. 58, 86 (1987).

[19] B. Nienhuis, in Phase Transitions and Critical Phenomena, edited by C. Domb and J. L. Lebowitz (Academic Press, London, 1987), Vol. 11. There is a typo in Eq. (4.26) of this work, describing the second magnetic exponent. In this formula, +1 has to be replaced by +2 .
[20] X. Qian, Y. Deng, and H. W. J. Blöte, Phys. Rev. E 72, 056132 (2005).

[21] B. Nienhuis and M. Nauenberg, Phys. Rev. Lett. 35, 477 (1975).

[22] The value $Q_{0}=q /(q+1)$ follows if, at coexistence, the $q$ magnetized phases are equally probable as the disordered phase. This value is confirmed by an exact asymptotic $L \rightarrow \infty$ analysis of finite Potts models in the mean-field limit $R \rightarrow \infty$.

[23] J. Lee and J. M. Kosterlitz, Phys. Rev. Lett. 65, 137 (1990).

[24] L. Mittag and M. J. Stephen, J. Phys. A 7, L109 (1974).

[25] F. Y. Wu, Rev. Mod. Phys. 54, 235 (1982).

[26] R. J. Baxter and F. Y. Wu, Phys. Rev. Lett. 31, 1294 (1973); Aust. J. Phys. 27, 357 (1974).

[27] G. Kamieniarz and H. W. J. Blöte, J. Phys. A 26, 201 (1993).

[28] W. Selke, J. Stat. Mech. (2007) P04008.

[29] V. Dohm, Phys. Rev. E 77, 061128 (2008).

[30] H. W. J. Blöte and Y. Deng, Phys. Rev. E 66, 066110 (2002).

[31] M. A. Novotny and H. G. Evertz, in Computer Simulation Studies in Condensed-Matter Physics VI, edited by D. P. Landau, K. K. Mon, and H.-B. Schüttler (Springer, Berlin, 1993), p. 188.

[32] Y. Deng, W.-A. Guo, J. R. Heringa, H. W. J. Blöte, and B. Nienhuis, Nucl. Phys. B 827, 406 (2010).

[33] C. Rebbi and R. H. Swendsen, Phys. Rev. B 21, 4094 (1980).

[34] H. W. J. Blöte and M. P. Nightingale, Physica A (Amsterdam) 112, 405 (1982).

[35] F. Igloi, J. R. Heringa, M. M. F. Philippens, A. Hoogland, and H. W. J. Blöte, J. Phys. A 25, 6231 (1992).

[36] P. W. Kasteleyn and C. M. Fortuin, J. Phys. Soc. Jpn. 26 (Suppl.), 11 (1969); C. M. Fortuin and P. W. Kasteleyn, Physica (Amsterdam) 57, 536 (1972). 